\title{
Earthquake-induced prompt gravity signals identified in dense array data in Japan
}

\author{
Masaya Kimura ${ }^{1 *} \mathbb{0}$, Nobuki Kame ${ }^{1}$, Shingo Watada ${ }^{1}$, Makiko Ohtani $^{2}$, Akito Araya ${ }^{1}$, Yuichi Imanishi ${ }^{1}$, \\ Masaki Ando ${ }^{3}$ and Takashi Kunugi ${ }^{4}$
}

\begin{abstract}
Earthquake ruptures cause mass redistribution, which is expected to induce transient gravity perturbations simultaneously at all distances from the source before the arrival of P-waves. A recent research paper reported the detection of such prompt gravity signals from the 2011 Tohoku-Oki earthquake by comparing observed acceleration waveforms and model simulations. The 11 observed waveforms presented in that paper recorded in East Asia shared a similar trend above the background seismic noise and were in good agreement with the simulations. However, the signal detection was less quantitative because the significance of the observed signals was not discussed and the waveforms at other stations in the region were not shown. In this study, similar trends were not observed in most of the data recorded near the stations used in the aforementioned study, suggesting that the reported signals were only local noises. We thus took a different approach to identify the prompt signals. We optimized the multi-channel data recorded by superconducting gravimeters, broadband seismometers, and tiltmeters. Though no signal was identified in the single-trace records, the stacked trace of the broadband seismometer array data in Japan showed a clear signal above the reduced noise level. The signal amplitude was $0.25 \mathrm{~nm} / \mathrm{s}^{2}$ for an average distance of $987 \mathrm{~km}$ from the event hypocenter. This detection was confirmed with a statistical significance of $7 \sigma$, where $\sigma$ is the standard deviation of the amplitude of the background noise. This result provided the first constraint on the amplitude of the observed prompt signals and may serve as a reference in the detection of prompt signals in future earthquakes.
\end{abstract}

Keywords: Earthquake-induced gravity perturbation, Transient deformation, Time variable gravity, 2011 Tohoku-Oki earthquake

\section{Introduction}

Compressional seismic waves radiating from an earthquake accompany density perturbations, which give rise to widespread transient gravity perturbations $\delta$, even ahead of the wave front. The interest in earthquakeinduced prompt gravity perturbations has increased in terms of both theoretical prediction and data signal detection with their potential for earthquake early warning (Harms et al. 2015; Harms 2016; Montagner et al. 2016; Heaton 2017; Vallée et al. 2017; Kimura 2018; Kimura and Kame 2019). In this paper, "prompt" denotes

\footnotetext{
*Correspondence: mkimura@eri.u-tokyo.ac.jp

${ }^{1}$ Earthquake Research Institute, The University of Tokyo, Yayoi, Bunkyo-ku,

Tokyo 113-0032, Japan

Full list of author information is available at the end of the article
}

the time period between the event origin time and the P-wave arrival time.

Study by Montagner et al. (2016) was the first to discuss prompt gravity signals in observed data. They searched for the signal from the $2011 \mathrm{Mw} 9.0$ TohokuOki earthquake in the records of a superconducting gravimeter (SG) at Kamioka (approximately $510 \mathrm{~km}$ from the epicenter) and five nearby broadband seismometers of the Full Range Seismograph Network of Japan (F-net). Though they failed to identify a prompt signal with an amplitude that was obviously above the background noise level, they found that the 30-s average value immediately before the P-wave arrival was more prominent than the noise level with a statistical significance greater than $99 \%$ (corresponding to approximately $3 \sigma$ if the background noise has a normal distribution, where $\sigma$ is the standard deviation of the noise). Based on this finding, 
they claimed the presence of a prompt gravity signal from the event. However, 99\% significance seems considerably low for definite signal detection because it means that one in hundred samples exceeds a reference level; this is too frequent to claim an anomaly in time series analysis.

Heaton (2017) replied to Montagner et al. (2016) with an objection that their data analysis did not include the appropriate response of the Earth. He pointed out that in the measurement of prompt gravity perturbations, the acceleration motion of the observational site $\ddot{\boldsymbol{u}}$ has to be considered because the gravimeter output $(\boldsymbol{a})_{z}$ is affected by $\ddot{\boldsymbol{u}}$, i.e., $(\boldsymbol{a})_{z}=(\delta \boldsymbol{g})_{z}-(\ddot{\boldsymbol{u}})_{z}$, where $(\boldsymbol{x})_{z}$ indicates the vertical component of vector $\boldsymbol{x}$ with upward being positive. He exemplified in a simple spherical Earth model that the Earth's motion due to prompt gravity perturbations mostly decreases the gravimeter's sensitivity.

Vallée et al. (2017) reported the detection of prompt gravity signals from the 2011 event based on both data analysis and theoretical modeling. From the records of regional broadband seismic stations in the Japanese islands and the Asian continent, they selected 11 waveforms based on the study's criteria. Nine waveforms among them showed a consistent visible downward trend starting from the origin time up to the respective P-wave arrival times (Figure 1 of Vallée et al. 2017). They then numerically simulated the prompt signals for the 11 stations considering the acceleration motion of the observational sites, i.e., a direct scenario based on Heaton (2017). To synthesize the sensor output $(\boldsymbol{a})_{z}$, they evaluated both the gravity perturbation $\delta \boldsymbol{g}$ and the ground acceleration $\ddot{\boldsymbol{u}}$ directly generated by $\delta \boldsymbol{g}$ in a semi-infinite flat Earth model. The 11 pairs of observed and synthetic waveforms showed similarities to one another (Figure 3 in Vallée et al. 2017). However, their signal detection was relatively less quantitative. In contrast to Montagner et al. (2016), they did not discuss the significance of the observed signals with respect to background noise. In addition, the 11 observational stations they used were only a small subset of the available approximately 200 stations.

In this study, we search for prompt gravity signals from the 2011 event using a quantitative approach. Initially, we note that observed waveforms at other stations near those Vallée et al. (2017) presented barely showed a similar trend beyond noise ("Local records near the reported stations" section). Our analyses thus rely not on simulated waveforms but rather mostly on data, and we optimize multi-channel data recorded by different instruments ("Data" section). We first analyze SG data at two stations ("Superconducting gravimeters" section), but signal detection was unsuccessful. Next, we analyze records of the dense arrays of F-net ("F-net broadband seismometers" section) and High Sensitivity Seismograph Network Japan (Hi-net) ("Hi-net tiltmeters"
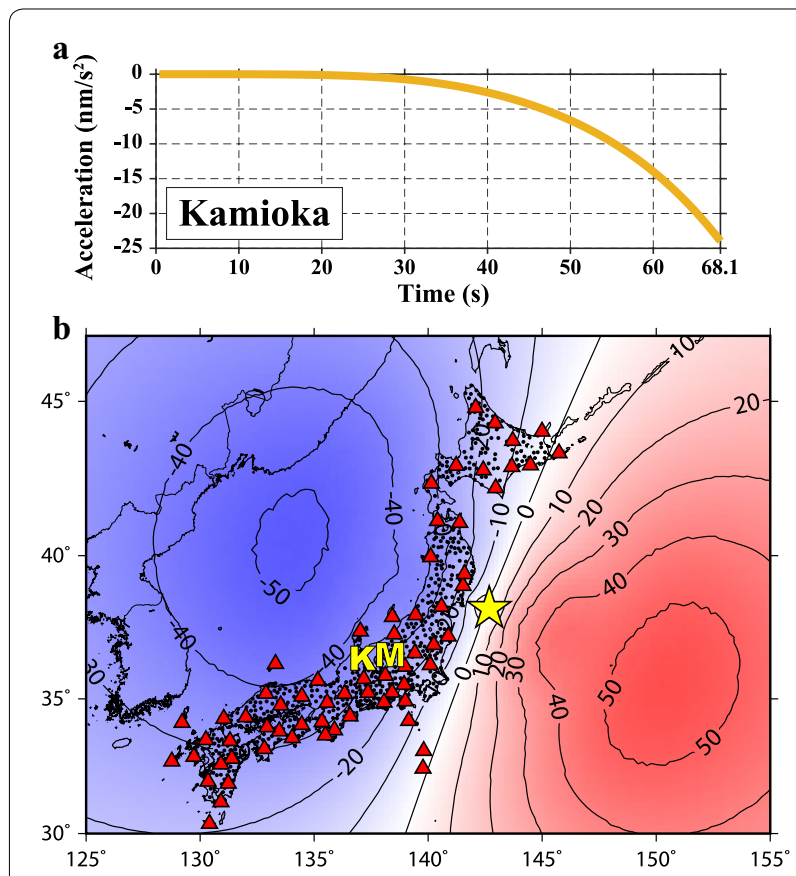

Fig. 1 a Model prediction of the prompt gravity perturbation $\left(\delta \boldsymbol{g}^{H}\right)_{z}$ (vertical component with upward positive) of the 2011 Tohoku-Oki earthquake for Kamioka Observatory. We used the infinite homogeneous Earth model of Harms et al. (2015), and no filter was applied. Time 0 was set to the event origin time teq. The P-wave arrival time on the gravimetric record is 05:47:32.4 UTC (68.1 s after $\left.t_{\mathrm{eq}}\right)$. $\mathbf{b}$ Distribution of the prompt gravity perturbation $\left(\delta \boldsymbol{g}^{\mathrm{H}}\right)$, immediately before P-wave arrival at each location. The contour lines are drawn every $10 \mathrm{~nm} / \mathrm{s}^{2}$. The star, the letters $\mathrm{K}$ and $\mathrm{M}$, the red triangle, and the small dots indicate the epicenter, Kamioka Observatory, Matsushiro Observatory, the 71 F-net stations, and the 706 tiltmeter stations, respectively

section). Although most single-channel records did not show any signal beyond noise, waveform-stacking successfully reduced the noise level and allowed identification of a prominent signal in the F-net data.

\section{Results of data analyses}

\section{Local records near the reported stations}

Vallée et al. (2017) selected 11 stations and showed the waveforms recorded at the stations. Their data processing (termed "procedure V" in this paper) and selection criteria are detailed in "Appendix 1." Because the presented waveforms showed a similar downward trend and amplitude in a wide range of hypocentral distance between 427 and $3044 \mathrm{~km}$, the prompt signal waveforms of the 2011 event are not expected to vary significantly within a few hundred kilometers. This long-range spatial characteristic is also supported by the original model of Harms et al. (2015), who formulated the prompt gravity perturbation $\delta \boldsymbol{g}^{\mathrm{H}}$ in an infinite homogeneous medium 
by an earthquake, where the superscript $H$ denotes the modeling by Harms et al. (2015). Figure 1 shows $\left(\delta \boldsymbol{g}^{\mathrm{H}}\right)_{z}$ for the 2011 event with contours drawn every $10 \mathrm{~nm} / \mathrm{s}^{2}$, often termed 1 micro gal in geodesy. The spatial extent of $\left(\delta \boldsymbol{g}^{\mathrm{H}}\right)_{z}$ is a few thousand kilometers (Fig. 1b) as noted by Kimura (2018).

We checked whether reported downward trends were recorded at other stations near those Vallée et al. (2017) used. Among the 11 stations, Fukue (FUK) in Japan and Mudanjiang (MDJ) and Zhalaiteqi Badaerhuzhen (NE93) in China had other available stations within $100 \mathrm{~km}$ and were eligible for this purpose.

Figure 2a (modified from Figure 3 of Vallée et al. 2017) shows the observed and simulated waveforms at FUK for reference, and Fig. $2 b$ shows the waveforms at the F-net stations near FUK. The hypocentral distances of FUK and the other 10 stations range from 1130 to $1390 \mathrm{~km}$ (Fig. 2c). The waveform at FUK (Fig. 2b) appears similar to that of Vallée et al. (2017) (Fig. 2a) as it shows a similar downward trend beyond the noise level with a similar amplitude. They are not identical to each other because of the different signal processing procedures of Vallée et al. (2017) (procedure V) and this study (termed "procedure K" in this paper). Details of procedure K and the difference in the two procedures are described in "Appendix 2."

However, the other 10 waveforms shown in Fig. $2 b$ do not generally depict a downward trend. Rather, they generally appear as only noise, although Sefuri (SBR) does seem to show a slight downward trend. Namely, the stations near FUK barely showed the downward trend as shown by the waveform at FUK.

Figure 3 shows the records at the stations surrounding MDJ and NE93 processed using procedure K. Again, similar downward trends are not observed at the stations near MDJ nor NE93, and it is difficult to identify a significant signal beyond noise in a single trace. Though the STS-1 broadband seismometer at MDJ shows the downward trend beyond noise, the other stations near MDJ, and the STS-2 broadband seismometer at MDJ, do not show such a signal. At NE93, not only the surrounding stations but also NE93 do not show the trend seen in Vallée et al. (2017).

Eventually, we did not see the downward trend except for a few outliers. This waveform comparison suggests that the downward trend at NE93 (Figure 1 of Vallée et al. 2017) was not a signal but an artifact due to procedure V,

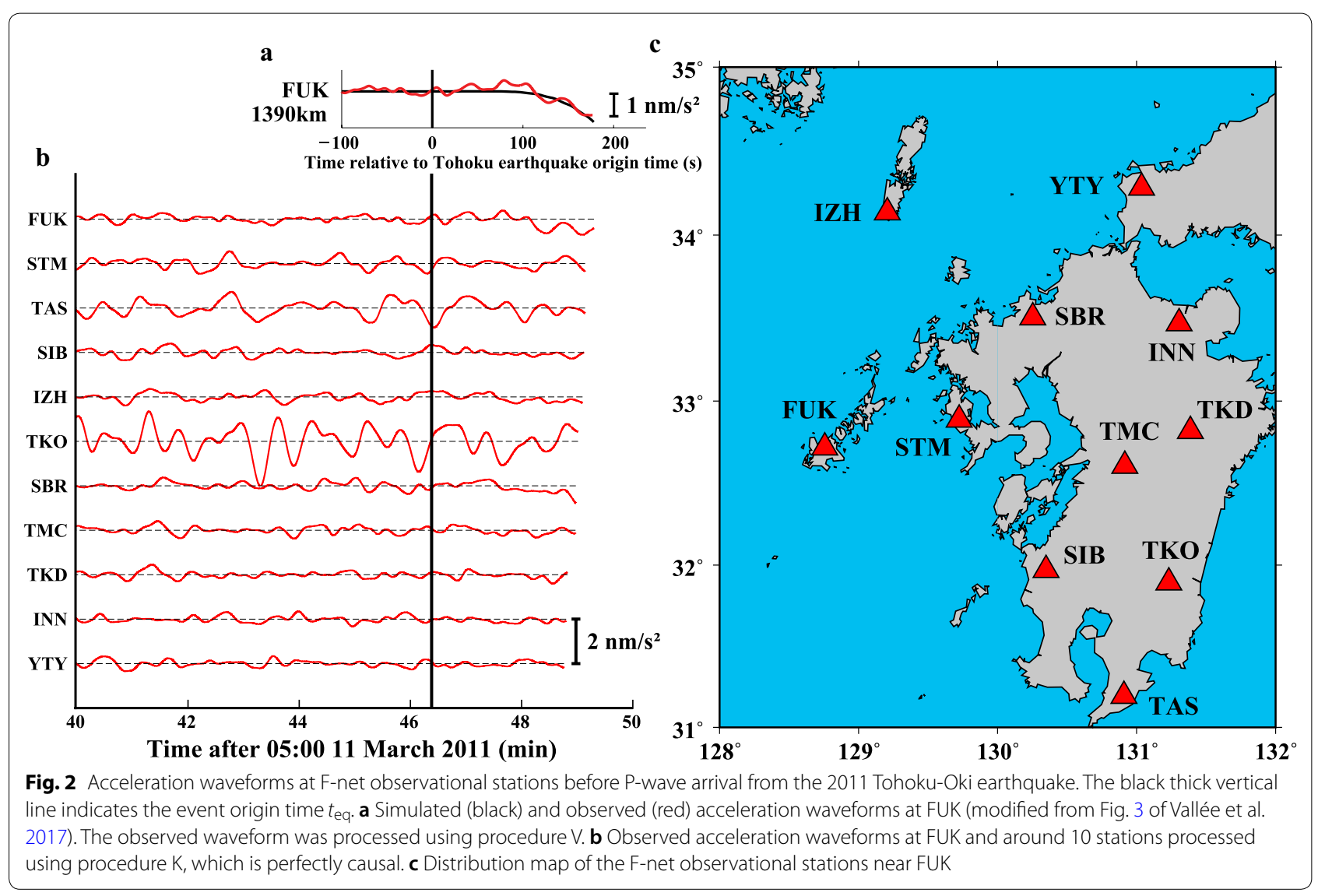




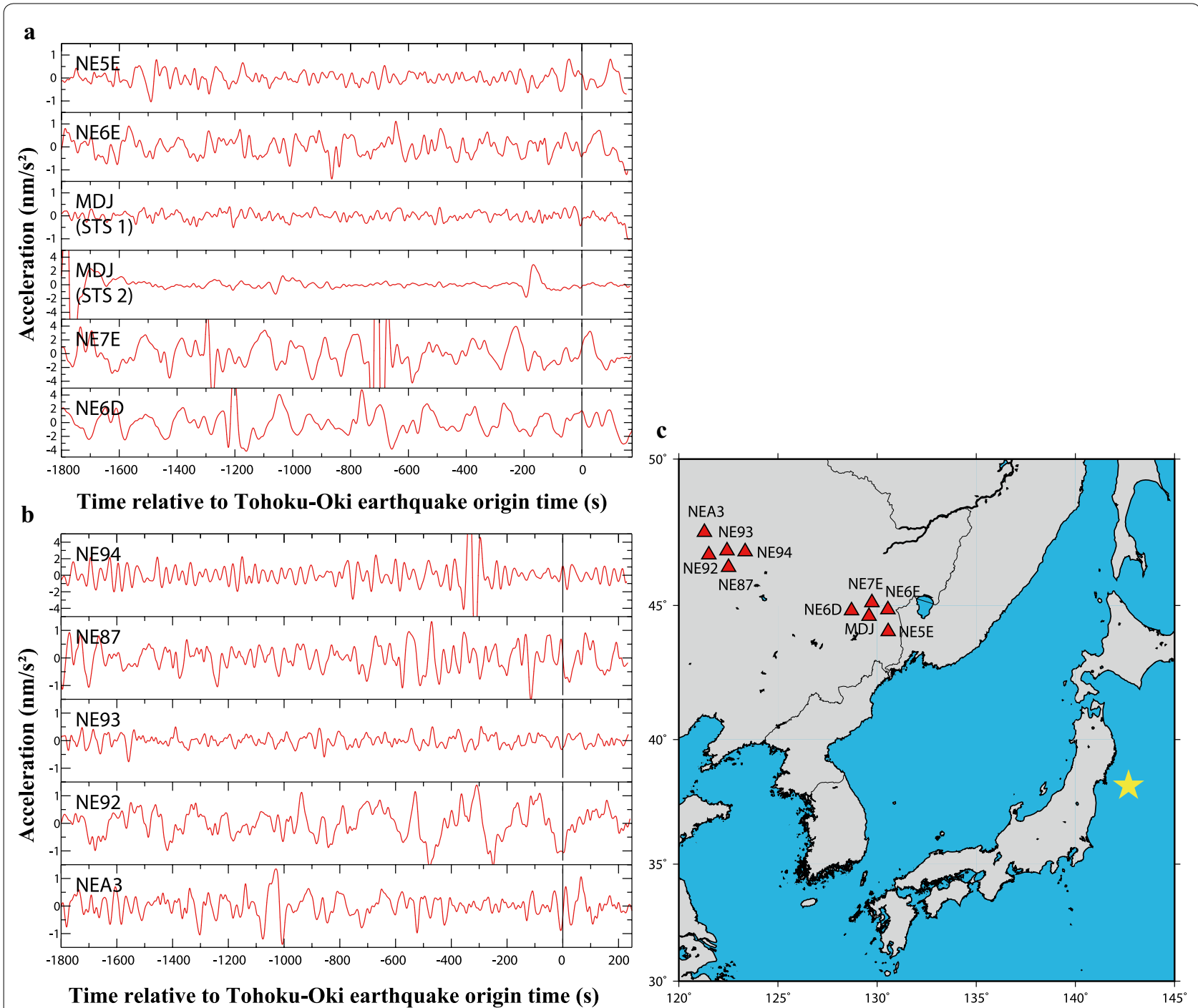

Fig. 3 Acceleration waveforms at observational stations in China before P-wave arrival from the 2011 Tohoku-Oki earthquake. The black vertical line indicates the event origin time, which was set to 0 . They were processed using procedure $\mathrm{K}$. a Observed acceleration waveforms at stations near MDJ: NE5E, NE6E, MDJ, NE7E, and NE6D. We plotted waveforms at MDJ for the STS-1 and STS-2 seismometers. Vallée et al. (2017) used data recorded by the STS-1 type. b Observed acceleration waveforms at stations near NE93: NE94, NE87, NE93, NE92, and NEA3. c Distribution map of the observational stations. The yellow star and red triangles indicate the epicenter and the stations, respectively

and the trend at FUK and MDJ was possibly just local site noise or affected by unknown local site responses.

\section{Data}

We analyzed three different types of data: gravity data from two SGs, ground velocity data from the F-net seismographic array, and ground tilt data from the $\mathrm{Hi}$ net tiltmeter array. All $71 \mathrm{~F}$-net stations are equipped with STS-1 or STS-2 broadband seismometers. A twocomponent borehole tiltmeter is installed at $706 \mathrm{Hi}$-net stations. These instruments are listed in Table 1 . The instrumental responses of SG, STS-1, and STS-2 to the acceleration input are shown in Additional file 1: Fig. S1.

\section{Superconducting gravimeters}

We used SG data recorded at a $40-\mathrm{Hz}$ sampling rate (GWR5 channel) (Imanishi 2001). Figure 4 shows the recorded data at Kamioka $\left(t_{\mathrm{P}}=t_{\mathrm{eq}}+68.1 \mathrm{~s}\right.$, where $t_{\mathrm{eq}}$ and $t_{\mathrm{P}}$ denote the event origin time and the visually selected P-wave arrival time, respectively). The data include the sensor response. The background microseism dominated, with an amplitude of $100 \mathrm{~nm} / \mathrm{s}^{2}$. Obviously, no signal was identified. Figure 5 shows the noise power spectrum. In contrast to the $1-\mathrm{Hz}$ sampling data (GGP1 channel) with a $0.061-\mathrm{Hz}$ anti-aliasing filter used in the analysis of Montagner et al. (2016), our 40- $\mathrm{Hz}$ sampling data contain the signal power in the frequency range 
Table 1 Observation instruments

\begin{tabular}{|c|c|c|c|c|}
\hline Instrument & Channels used & $\begin{array}{l}\text { Sampling } \\
\text { rate }(\mathrm{Hz})\end{array}$ & Distance from the hypocenter & Output used \\
\hline Superconducting gravimeter & 2 (Kamioka and Matsushiro) & 40 & $\begin{array}{l}515 \text { km (Kamioka), } \\
436 \text { km (Matsushiro) }\end{array}$ & Vertical gravity acceleration $\left(\mathrm{m} / \mathrm{s}^{2}\right)$ \\
\hline Broadband seismometer & 27 out of 71 (F-net) & 20 & From 505 to 1421 km & $\begin{array}{l}\text { Vertical ground velocity }(\mathrm{m} / \mathrm{s}) \\
\text { converted to acceleration }\left(\mathrm{m} / \mathrm{s}^{2}\right)\end{array}$ \\
\hline Tiltmeter & 553 out of 1412 (Hi-net) & 20 & From 264 to 1349 km & $\begin{array}{l}\text { Ground tilt (rad) converted to } \\
\text { horizontal acceleration }\left(\mathrm{m} / \mathrm{s}^{2}\right)\end{array}$ \\
\hline
\end{tabular}

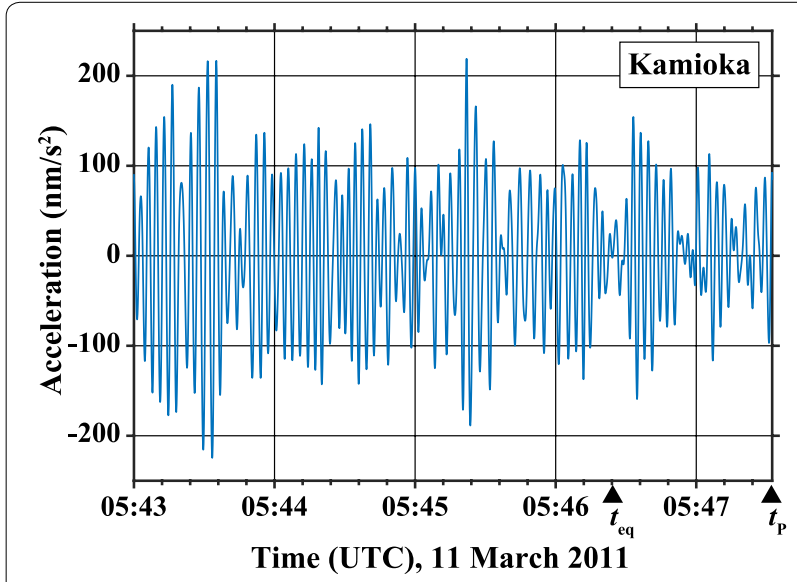

Fig. 4 Original SG data at Kamioka with zero direct current offset (at a $40-\mathrm{Hz}$ sampling rate)

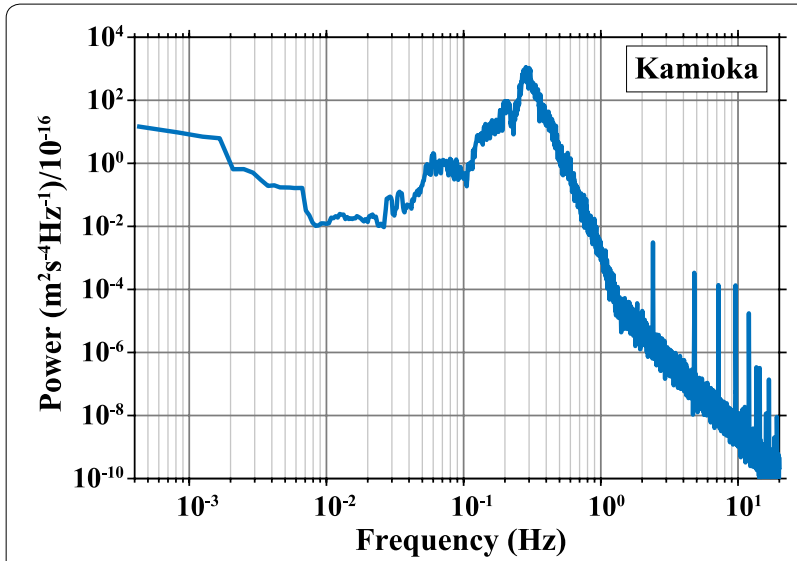

Fig. 5 Noise power spectrum of the Kamioka SG data. The time window is 40 min between 05:00 and 05:40 UTC before the 2011 Tohoku-Oki event

higher than $0.061 \mathrm{~Hz}$. After removing the trend component and multiplying a cosine taper in the first and last $10 \%$ sections of the time series, we applied a band-pass filter (five-pole $0.001-\mathrm{Hz}$ high-pass and five-pole 0.03$\mathrm{Hz}$ low-pass causal Butterworth filters) to the 1-h data
(05:00-06:00 UTC) to reduce the relatively large noise power higher than $0.05 \mathrm{~Hz}$. The lower corner frequency of $0.001 \mathrm{~Hz}$ was set to remove the long period tidal variation. After filtering, the noise was significantly reduced (Fig. 6a). During the prompt period $t_{\mathrm{eq}}<t<t_{\mathrm{P}}$, we do not see signals with amplitudes far beyond the noise level of the record prior to the event origin time.

For quantitative evaluation, we defined the noise level $A_{\mathrm{N}}$ in the time window $\left[t_{1}, t_{2}\right]$ as follows:

$$
A_{\mathrm{N}}=\sqrt{\frac{1}{t_{2}-t_{1}} \int_{t_{1}}^{t_{2}}[x(t)-\mu]^{2} \mathrm{~d} t}
$$

where $x(t)$ is time series data and $\mu=\frac{1}{t_{2}-t_{1}} \int_{t_{1}}^{t_{2}} x(t) \mathrm{d} t$. For the Kamioka data, $A_{\mathrm{N}}$ decreased from 70 to $0.4 \mathrm{~nm} / \mathrm{s}^{2}$ after filtering $\left(t_{1}=05: 40 \mathrm{UTC}\right.$ and $\left.t_{2}=t_{\mathrm{eq}}\right)$.

Figure $6 \mathrm{~b}$ shows the data for Matsushiro $(436 \mathrm{~km}$ from the hypocenter and $t_{\mathrm{P}}=t_{\mathrm{eq}}+57.3 \mathrm{~s}$ ) after the same filtering process. Although $A_{\mathrm{N}}$ decreased from 80 to $0.7 \mathrm{~nm} / \mathrm{s}^{2}$ after filtering, we did not recognize clear signals during the prompt period. Note that the oscillation with the period of approximately $90 \mathrm{~s}$ is a parasitic mode of the instrument (Imanishi 2005, 2009).

\section{F-net broadband seismometers}

The frequency responses of the F-net STS-1 seismometers to velocity are flat between 0.003 and $10 \mathrm{~Hz}$. Consequently, we did not deconvolve the sensor frequency responses from the recorded waveforms. The velocity data were converted to acceleration data taking the finite difference in the time domain.

In the vertical component of the F-net data, the typical value of $A_{\mathrm{N}}$ was $1000 \mathrm{~nm} / \mathrm{s}^{2}\left(340 \mathrm{~nm} / \mathrm{s}^{2}\right.$ was the lowest value) dominated by the microseism. To reduce the microseismic noise, we applied the same filters $(0.002-\mathrm{Hz}$ two-pole high-pass and $0.03-\mathrm{Hz}$ six-pole low-pass causal Butterworth filters) employed in Vallée et al. (2017) for all available data from 70 of the 71 stations (omitting one because of the poor recording quality). After filtering, the 


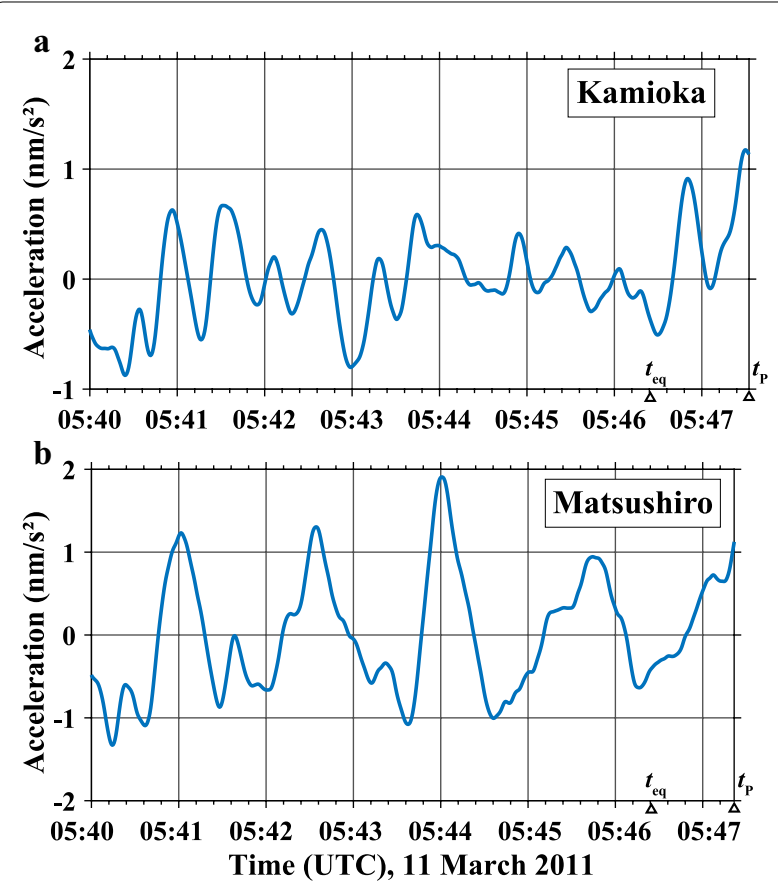

Fig. $60.001-0.03 \mathrm{~Hz}$ band-pass-filtered SG data at a Kamioka and b Matsushiro

microseism noise was successfully reduced to as low as $0.2 \mathrm{~nm} / \mathrm{s}^{2}$ (Additional file 1: Fig. S2). However, we did not recognize clear signals. Only at two stations, FUK and SBR, could we find a downward trend before P-wave arrival.

Next, a multi-station signal-stacking method was applied to further enhance the signals of interest. After the band-pass filtering, we selected 27 traces out of the 70 traces based on the noise level and stacked them aligned with $t_{\mathrm{P}}$ at each station because we expected the maximum signal amplitude at the last of the prompt period (Fig. 1a). Figure 7a shows the stacked trace, and Additional file 1: Fig. S3a shows an enlarged view of the trace. The noise of the stacked trace significantly decreased, and the trace successfully showed a significant signal with an amplitude of $0.25 \mathrm{~nm} / \mathrm{s}^{2}$. Our selection criterion and polarity reversal correction for the stacking are described in "Appendix 3," and the 27 stations are listed in Additional file 2: Table S1. The hypocentral distances of the 27 stations are between 505 and $1421 \mathrm{~km}$ (the average is $987 \mathrm{~km}$ ), and the minimum and maximum $t_{\mathrm{P}}$ are 63 and $176 \mathrm{~s}$ after $t_{\text {eq }}$, respectively.

To quantify the signal detection in terms of statistical significance, we investigated the distributions of background noise and the enhanced gravity signal. Figure 8 shows the histograms of the noise section (between -30 and -3 min before the aligned $t_{\mathrm{P}}$ ) and the signal section in the stacked trace. Here, we defined the latter half of the time period $-1 \min \left(\fallingdotseq \operatorname{minimum} t_{\mathrm{P}}-t_{\mathrm{eq}}\right)<t<0$, i.e.,

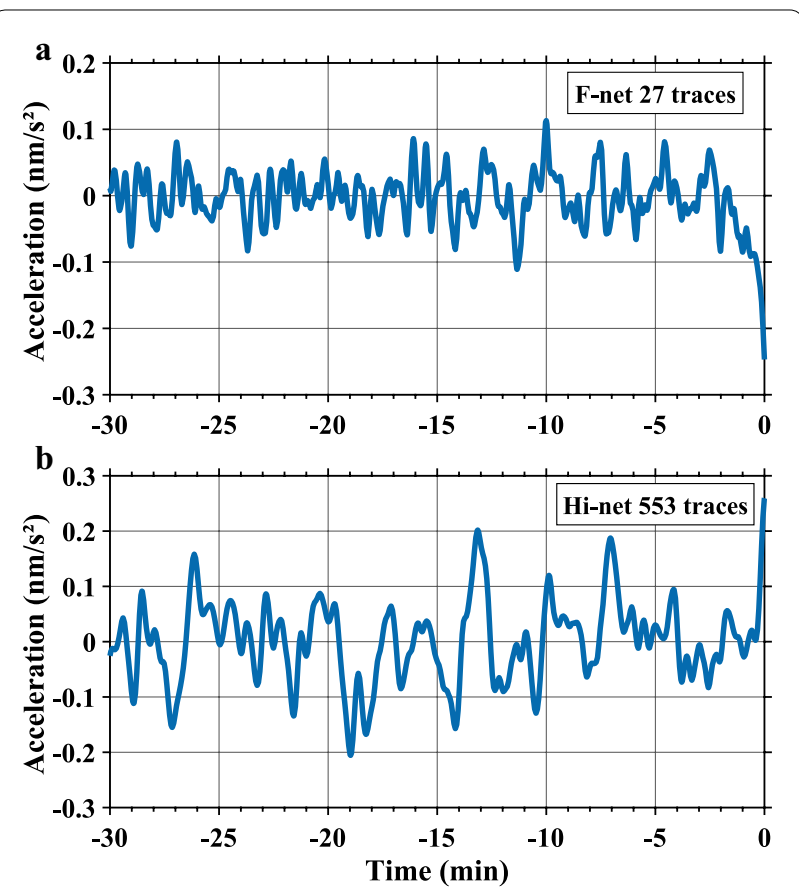

Fig. 7 Stacked waveforms of the filtered data for 30 min before the P-wave arrivals. Time 0 was set to the stacking reference time $t_{p}$. a Plot for F-net broadband seismometer data. b Plot for Hi-net tiltmeter data

$-30 \mathrm{~s}<t<0$ as the signal section because all 27 waveforms were expected to contain a signal with increasing amplitude toward the end of this time period, as shown in Fig. 1a. The noise histogram was approximated by a normal distribution with a standard deviation $\sigma$. In our analysis, $\sigma$ was given by $A_{\mathrm{N}}\left(0.035 \mathrm{~nm} / \mathrm{s}^{2}\right)$. On the other hand, before the aligned $t_{\mathrm{P}}$, the amplitude of the stacked trace generally increased with time and the signal level exceeded $3 \sigma$ at $t=-20 \mathrm{~s}$ and $5 \sigma$ at $t=-6 \mathrm{~s}$ before finally reaching $7 \sigma$ at $t=0$ (Fig. 7a), i.e., the signal detection was verified with a statistical significance of $7 \sigma$.

\section{Hi-net tiltmeters}

We also analyzed the data recorded by the Hi-net tiltmeters, which work as horizontal accelerometers. For our analysis, the tilt data in rad were converted into horizontal acceleration in $\mathrm{m} / \mathrm{s}^{2}$ by multiplying with the gravity acceleration $\left(9.8 \mathrm{~m} / \mathrm{s}^{2}\right)$. Because the sensor response is not known in the seismic frequency band, we could not deconvolve it from the data; however, tiltmeter records have been used as seismic records by comparing them to nearby broadband seismic records (e.g., within a bandwidth of $0.02-0.16 \mathrm{~Hz}$ ) (Tonegawa et al. 2006). Because tiltmeters are designed to respond to static changes, recordings are also reliable below $0.02 \mathrm{~Hz}$. 


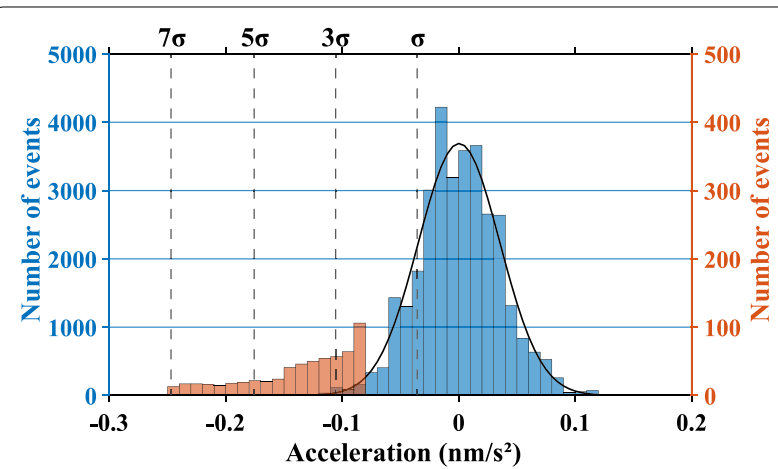

Fig. 8 Amplitude histograms of the background noise (blue in the left vertical axis) and the prompt gravity signal before P-wave arrival (red in the right vertical axis). The noise histogram was fitted by a normal distribution with a standard deviation $\sigma=0.035 \mathrm{~nm} / \mathrm{s}^{2}$ (black curve)

When compared to the F-net data, the Hi-net tiltmeter data were generally noisy. The typical value of $A_{\mathrm{N}}$ was $2000 \mathrm{~nm} / \mathrm{s}^{2}$. After removing the trend component and applying the same band-pass filter as employed in Vallée et al. (2017), we again failed to identify a significant signal in each channel. We then aligned 553 data traces out of 1412 traces (two horizontal components from each station) with respect to the P-wave arrival times for stacking. Our selection criterion is also described in "Appendix 3." The hypocentral distances of the 553 traces are between 264 and $1349 \mathrm{~km}$ (the average is $830 \mathrm{~km}$ ). Figure $7 \mathrm{~b}$ and Additional file 1: Figure S3b show the stacked trace and its enlarged view, respectively. In contrast to the F-net results, the prompt signal was not identified. The noise level $A_{\mathrm{N}}$ was $0.08 \mathrm{~nm} / \mathrm{s}^{2}$.

The predicted signal amplitude of the stacked trace based on the infinite homogeneous Earth model of Harms et al. (2015) was $2 \mathrm{~nm} / \mathrm{s}^{2}$ (Kimura 2018), where the theoretical time series were synthesized at each station and then filtered and stacked in alignment with the $\mathrm{P}$-wave arrival time in the same manner as the observed data. In our analysis, such a large signal was confirmed not to exist in the data, and the upper signal level was constrained as $0.15 \mathrm{~nm} / \mathrm{s}^{2}$ with $95 \%$ statistical significance (approximately $2 \sigma$ ) in the horizontal component.

\section{Discussion}

\section{Difference from previous SG study}

Failure of detecting prompt gravity signals in the SG data is consistent with the result of Montagner et al. (2016), who also analyzed the Kamioka SG and five nearby F-net stations and could not visually detect a clear signal in the time domain. On the other hand, at the Global Seismographic Network (GSN) station Matsushiro (MAJO), a signal detection was reported by Vallée et al. (2017). GSN
MAJO and the Matsushiro SG are installed in the same tunnel, and the Kamioka SG and the five nearby F-net stations in Montagner et al. (2016) are located at nearly the same epicentral distance. The results of Montagner et al. (2016) and Vallée et al. (2017) seem inconsistent with one another. The signal amplitude at GSN MAJO shown in Vallée et al. (2017) may have been a mere noise or affected by a local site response.

\section{Significance of our stacked trace on theoretical modeling}

The F-net stacked trace (Fig. 7a) showed a great improvement of the statistical significance of the signal detection. It provides the first constraint of prompt gravity signals by observation and can work as a reference to validate future theoretical models. Once a model is developed that explains the sensor output in gravimetry and the reliable value of $\delta \boldsymbol{g}$ is constrained, related physical quantities such as gravity gradient and spatial strain are constrained as well.

As Heaton (2017) noted, ground acceleration $\ddot{\boldsymbol{u}}$ affects the measurement of prompt gravity perturbation $\delta \boldsymbol{g}$. Therefore, in the modeling of prompt gravity signals, not only $\delta \boldsymbol{g}$ but also $\ddot{\boldsymbol{u}}$ before P-wave arrival have to be calculated. Vallée et al. (2017) analytically showed that in an infinite homogeneous non-self-gravitating medium, the induced $\ddot{\boldsymbol{u}}$ directly generated by $\delta \boldsymbol{g}$ becomes $\ddot{\boldsymbol{u}}=\delta \boldsymbol{g}$, suggesting full cancelation of $\delta \boldsymbol{g}$ by $\ddot{\boldsymbol{u}}$. They then numerically investigated $\delta \boldsymbol{g}$ and the induced site motion $\ddot{\boldsymbol{u}}$ when exposed to the effects of a free surface in a layered nonself-gravitating half-space and evaluated the sensor output $-(\delta \boldsymbol{g})_{z}+(\ddot{\boldsymbol{u}})_{z}$. Their simulated waveforms at the 11 stations showed the same downward monotonic trend and similar amplitude of approximately $1 \mathrm{~nm} / \mathrm{s}^{2}$ within the wide range of 427-3044 $\mathrm{km}$ from the hypocenter.

However, this simulated signal amplitude of $1 \mathrm{~nm} /$ $\mathrm{s}^{2}$ is significantly larger than our identified amplitude of $0.25 \mathrm{~nm} / \mathrm{s}^{2}$ in the F-net stacked trace, suggesting that the simulation of Vallée et al. (2017) overestimated the sensor outputs. Our stacked waveform and Vallée et al.s single-channel waveforms cannot be directly compared, but their amplitudes can be compared. Because all 27 stations used for the stacking are in the region where Vallée et al's simulated waveforms showed the same trend and amplitude of $1 \mathrm{~nm} / \mathrm{s}^{2}$, if similar signals were recorded in the 27 traces, the resultant amplitude of the stacked waveform would also become $1 \mathrm{~nm} / \mathrm{s}^{2}$. The identified signal level of $0.25 \mathrm{~nm} / \mathrm{s}^{2}$ is, however, one-fourth of the expected value. Notably, the polarity of our stacked trace shows a negative trend toward the P-wave arrival, consistent with the observation and simulation of Vallée et al. (2017).

A prospective candidate for a better theoretical model is a normal mode model of a spherical self-gravitating realistic Earth that addresses the fully coupled 
equations between the elastic deformation and gravity. Very recently, Juhel et al. (2019) conducted theoretical modeling using such a normal mode approach to compute prompt gravity signals. However, similar to Vallée et al. (2017), the fully coupled problem was not solved in the study. They first considered the prompt gravity perturbation $\delta \boldsymbol{g}$ induced by the earthquake elastic deformation and then considered the prompt gravity effect on the elastic deformation, which they termed a "two-step approach." Their simulation results were quite similar to those of Vallée et al. (2017) and seemed to overestimate the sensor output as well. Although a fully coupled model

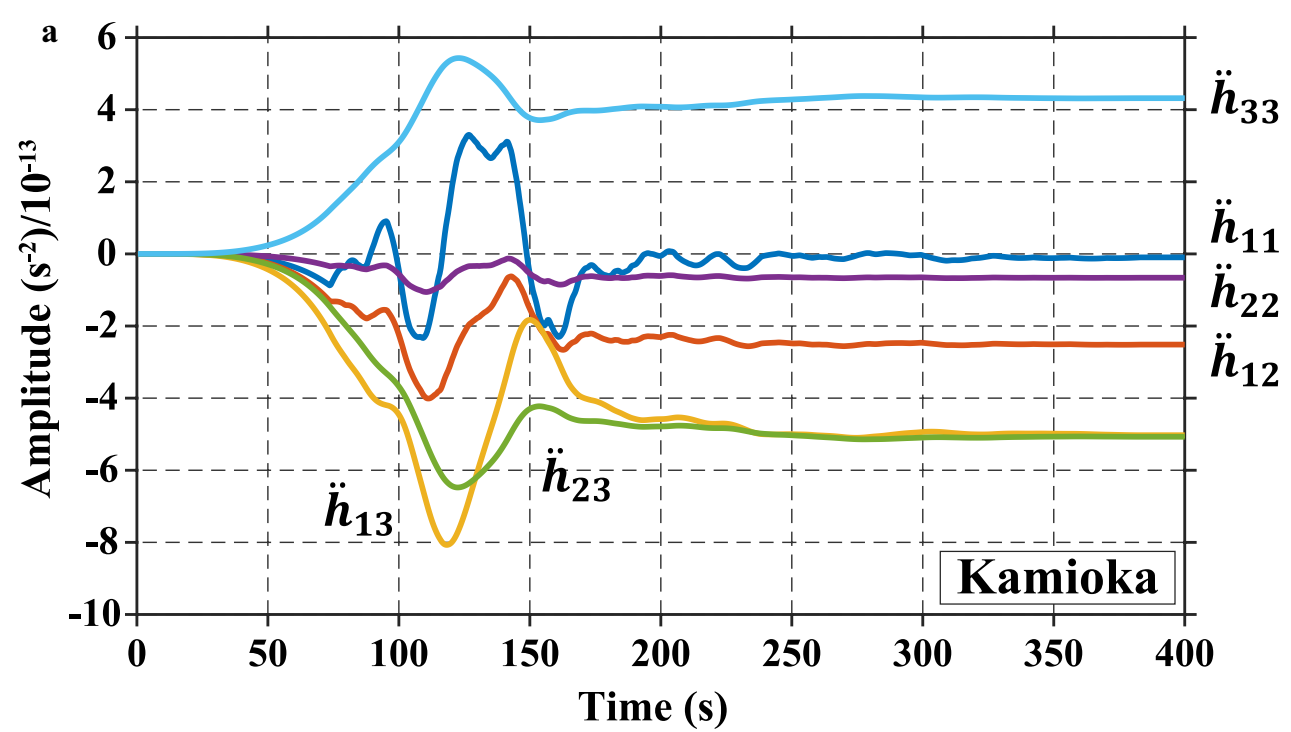

b
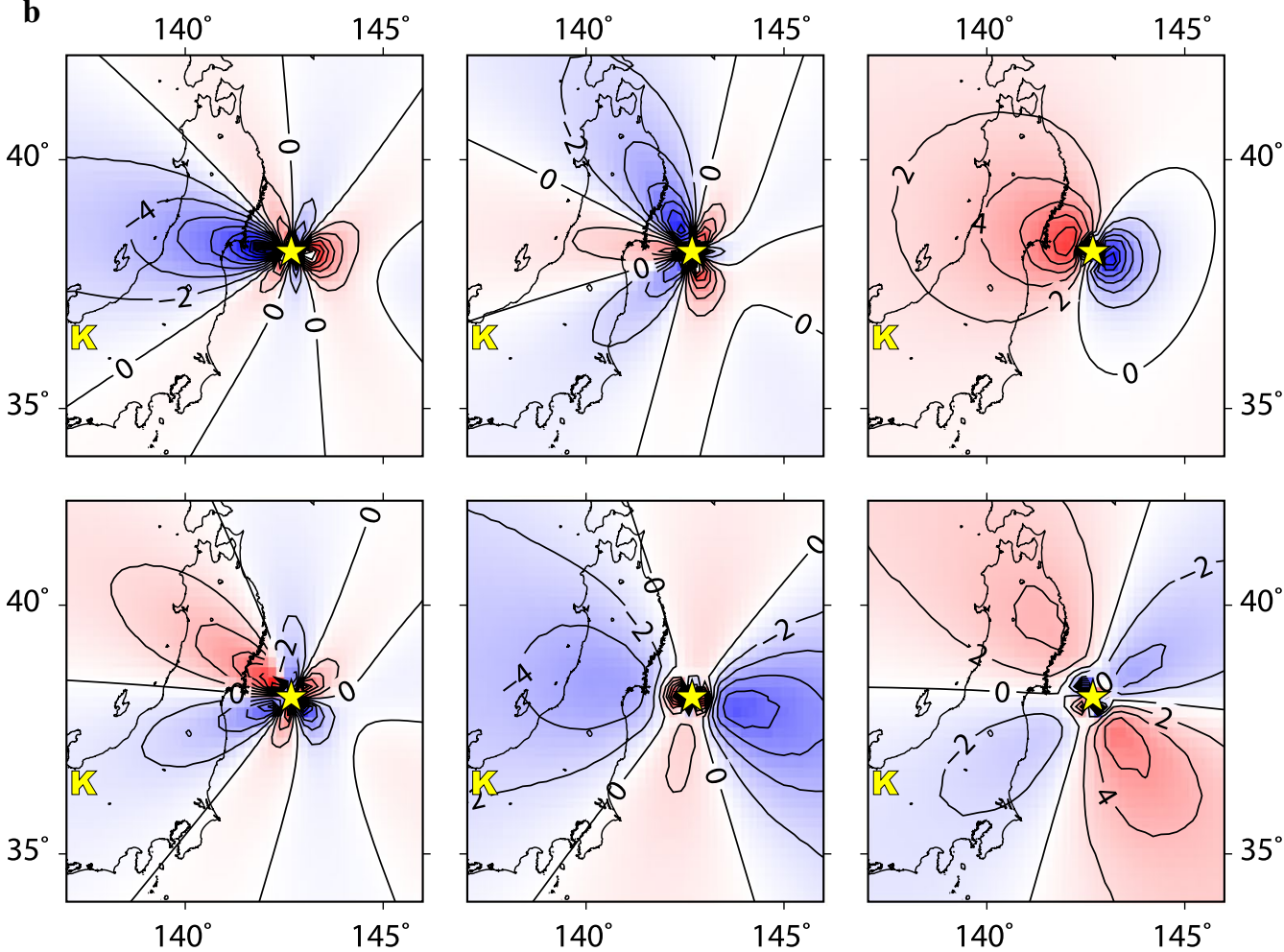

Fig. 9 a Theoretical six-component gravito-gradiograms of the 2011 Tohoku-Oki earthquake synthesized for Kamioka Observatory. Time 0 was set to the event origin time $t_{\text {eq. }}$ b Distribution of prompt gravity gradient changes immediately before P-wave arrival at each location (upper left: $\ddot{h}_{11}$ component, upper center: $\ddot{h}_{22}$ component, upper right: $\ddot{h}_{33}$ component, lower left: $\ddot{h}_{12}$ component, lower center: $\ddot{h}_{13}$ component, and lower right: $\ddot{h}_{23}$ component), where $\ddot{h}_{i j}$ denotes the $i j$ th component of the gravity gradient tensor (see "Appendix $4^{\prime \prime}$ ). In these figures, the $x_{1^{-}}, x_{2^{-}}$, and $x_{3}$ -axes correspond to the directions of east, north, and upward, respectively. The star and the letter $\mathrm{K}$ are the epicenter and Kamioka Observatory, respectively. The contour lines are drawn every $2 \times 10^{-13} \mathrm{~s}^{-2}$ 
requires an enormous number of normal mode summations to precisely evaluate the prompt gravity perturbations, a numerical assessment should be conducted in the future.

\section{Possible reasons for no finding with tiltmeters}

The lack of signal identification in the stacked Hi-net trace (Fig. 7b) can be attributed to the large amplitude of the noise spectrum in the frequency band of the applied band-pass filter (in contrast to the SG and the F-net data in the vertical component). In this band, the typical noise level is more than 10 times that of the quiet SGs and the F-net. Another reason may be unknown effects of a free surface on the induced $\ddot{\boldsymbol{u}}$, which may more effectively cancel the horizontal component of $\delta g$ compared to its vertical component.

\section{Toward future detection of earthquake-induced prompt gravity signals using a gravity gradient sensor}

We have shown that the identified prompt gravity signals were very small (approximately $0.25 \mathrm{~nm} / \mathrm{s}^{2}$ for the average distance of $987 \mathrm{~km}$ ); this can be attributed to the cancelation of gravity measurements by the acceleration motion of the ground and suggests that gravimetry is not the best approach for detecting prompt gravity perturbation. A gravity gradient measurement provides an alternative method to detect prompt signals from earthquakes (Harms et al. 2015; Juhel et al. 2018). A spatially inhomogeneous gravity field induces tidal deformation of an object or spatial strain, which is observable even if the observer moves with the same acceleration as the prompt gravity perturbation.

Detecting very small perturbations in the gravity gradient has been a challenge in identifying gravitational waves from space. Abbott et al. (2016) observed gravitational waves using laser interferometers in a highfrequency range from tens to hundreds of $\mathrm{Hz}$. New state-of-the-art instruments, such as torsion bar antennas (TOBA) (Ando et al. 2010; Shoda et al. 2014), are being developed. Such instruments are intended to observe spatial strain through the tidal deformation of two crossing bars. The existing prototype TOBA attained a $10^{-8} \mathrm{~s}^{-2}$ sensitivity within a low-frequency range of 0.01-1 Hz (Shoda et al. 2014). The theoretical gravitogradiograms and the prompt signal intensity map are shown for the 2011 Tohoku-Oki earthquake (Fig. 9). The expected signal level was $10^{-13} \mathrm{~s}^{-2}$. Though this value is $10^{-5}$ times smaller than the attained sensitivity, the next-generation TOBA will attain sufficient sensitivity to detect prompt signals. Prompt earthquake detection will significantly benefit from such ultra-sensitive sensors.

In "Appendix 4," we present an explicit expression of theoretical gravito-gradiograms, the waveforms of gravity gradients. We extended the expression of Harms et al. (2015), who used a seismic dislocation source, to a general source described as a moment tensor. Our extension will contribute to the interpretation of future observational records of various event mechanisms.

\section{Conclusions}

We searched for prompt gravity signals from the 2011 Mw 9.0 Tohoku-Oki earthquake in seismic network data. Though nearly all the single-channel waveforms did not show any signals beyond the noise level except for several outliers, the stacked trace of F-net broadband records showed a clear signal in the vertical component. The identified signal level was $0.25 \mathrm{~nm} / \mathrm{s}^{2}$ for the average distance of $987 \mathrm{~km}$; this detection was verified at a statistical significance of $7 \sigma$ to the background noise. In addition, analysis of Hi-net tiltmeters constrained the upper limit of the signal in the horizontal components as $0.15 \mathrm{~nm} /$ $\mathrm{s}^{2}$ at $95 \%$ significance. The stacked F-net trace is the first constraint of earthquake-induced prompt gravity signals by observation and will be used as a reference to validate future theoretical models. Measurement of gravity gradients is a more promising method in the prompt detection of future earthquakes. State-of-the-art instruments, such as torsion bar antennas, are being developed to detect strain acceleration smaller than $10^{-13} \mathrm{~s}^{-2}$.

\section{Additional files}

Additional file 1. Figures showing (1) a diagram of instrumental responses, (2) acceleration waveforms of F-net broadband seismometers, and (3) an enlarged view of the stacked traces of F-net broadband seismometers and Hi-net tiltmeters data.

Additional file 2. List of the 27 F-net stations used for the stacking.

\section{Abbreviations}

FUK: Fukue; F-net: Full Range Seismograph Network of Japan; GSN: Global Seismographic Network; Hi-net: High Sensitivity Seismograph Network Japan; IRIS: Incorporated Research Institutions for Seismology; MAJO: Matsushiro; MDJ: Mudanjiang; NE93: Zhalaiteqi Badaerhuzhen; SAC: Seismic Analysis Code; SBR: Sefuri; SG: superconducting gravimeter; TOBA: torsion bar antennas.

\section{Authors' contributions}

MK performed most of the waveform analysis. NK supervised MK's work. NK and MK wrote the manuscript. MK, NK, and SW contributed to the planning. NK, SW, MO, AA, YI, MA, and TK contributed to the interpretation of the results and gave useful advice. YI and TK contributed to the SG and Hi-net tiltmeter data acquisition, respectively. All authors read and approved the final manuscript.

\section{Author details}

1 Earthquake Research Institute, The University of Tokyo, Yayoi, Bunkyo-ku, Tokyo 113-0032, Japan. ${ }^{2}$ National Institute of Advanced Industrial Science and Technology, Namiki, Tsukuba 305-8560, Japan. ${ }^{3}$ Department of Physics, The University of Tokyo, Hongo, Bunkyo-ku, Tokyo 113-0033, Japan. ${ }^{4}$ National Research Institute for Earth Science and Disaster Resilience, Tennodai, Tsukuba 305-0006, Japan. 
Acknowledgements

We thank the editor Severine Rosat and three anonymous reviewers for their constructive comments and suggestions that helped to improve the manuscript. We also thank Nobuaki Fuji for valuable discussion.

\section{Competing interests}

The authors declare that they have no competing interests.

\section{Availability of data and materials}

The SG data used in our study are available on request from the authors. The F-net data are available at NIED F-net server http://www.fnet.bosai.go.jp. The Hi-net tiltmeter data used in our study can be obtained from NIED Japan by sending a request to hinet-admin@bosai.go.jp.

Consent for publication

Not applicable.

Ethics approval and consent to participate

Not applicable.

Funding

This research was supported by JSPS (KAKENHI JP15K13559, JP16K05532, JP18J21734) and MEXT via the Program for Leading Graduate Schools and the Earthquake and Volcano Hazards Observation and Research Program.

\section{Appendix 1: Data processing and station selection criteria of Vallée et al. (2017) and characteristics of the waveforms presented in the study}

Vallée et al. (2017) retrieved all the regional broadband vertical seismic records at distances up to $3000 \mathrm{~km}$ from the 2011 Tohoku-Oki earthquake hypocenter from the Incorporated Research Institutions for Seismology (IRIS) data center and from F-net. The number of stations was approximately 200 in this region, many of which were deployed in Japan and northeast China. They conducted signal processing (procedure $\mathrm{V}$ ) as follows: they (1) terminated each station time series at the P-wave arrival time; (2) removed the mean value; (3) deconvolved the sensor response and converted it into a band-limited accelerogram using the Seismic Analysis Code (SAC, Goldstein and Snoke 2005) command "transfer"; and then (4) applied a band-pass filter $(0.002-\mathrm{Hz}$ two-pole high-pass and $0.03-\mathrm{Hz}$ six-pole low-pass causal Butterworth filters). During procedure $\mathrm{V}$, no tapering was applied to the records.

Among all the processed records, they selected nine records based on a noise criterion that the waveform amplitude never exceeded $\pm 0.8 \mathrm{~nm} / \mathrm{s}^{2}$ during the 30-min window before the earthquake origin time. They additionally selected two F-net stations (Shari, Fukue) to improve the azimuthal and distance coverage. The hypocentral distances of the selected 11 stations ranged from 427 to $3044 \mathrm{~km}$.

Nine waveforms out of the 11 , three in the Japanese islands and six in the Asian continent, shared a downward trend beyond the seismic noise before the $\mathrm{P}$-wave arrivals. The amplitudes immediately prior to the $\mathrm{P}$-wave arrivals were approximately $1 \mathrm{~nm} / \mathrm{s}^{2}$ for the hypocentral distance 1000-2000 km (Fig. 1 of Vallée et al. 2017), in which Vallée et al. considered the observability of the signals reaches a maximum.

\section{Appendix 2: Data processing of this study and the difference between Vallée et al.'s procedures and those of this study}

Our data processing (procedure K) is as follows: we (1) extracted the 60-min time series data starting at $46 \mathrm{~min}$ before the origin time; (2) calibrated the raw digital count data into velocity by dividing by the sensor sensitivity coefficient; (3) converted it from velocity to acceleration through the finite difference of digital velocity data; (4) multiplied a cosine taper at the first and last $10 \%$ sections of the time series; and then (5) applied the same bandpass filter employed in procedure V. The 60-min time series are sufficiently long to avoid the taper to decrease the signal of interest.

Procedure $\mathrm{K}$ does not involve the recovery of the instrumental response and therefore is perfectly causal. Though we used data containing the following large amplitude of the P-waves, non-causal artificial signals never originated from the section. In contrast, as mentioned in "Appendix 1", Vallée et al. (2017) terminated the waveforms at the P-wave arrival time and deconvolved the sensor response. Removal of the instrumental response from the terminal portion of time series data works as an acausal filter for the waveform, which can generate spurious signals just before P-wave arrival, as exemplified in Fig. 3b, in which the retrieved waveforms were processed using our perfectly causal method. The waveform at NE93 does not show the downward trend seen in Vallée et al. (2017).

Note that most of the available data were clipped after the P-wave arrivals. That is why both Vallée et al. (2017) and our study did not deconvolve the sensor response from the time series including the P-wave section.

\section{Appendix 3: Station selection criterion and polarity reversal correction for stacking of F-net and Hi-net data}

For the stacking of F-net data, we selected 27 stations where the noise level $A_{\mathrm{N}}$ was less than one-twentieth of the reference value $A_{\mathrm{S}}$. Here, $A_{\mathrm{S}}$ is the amplitude of the synthetic gravity waveforms predicted by Harms et al. (2015) and applied the same filter as for the observed waveforms. As an example, the filtering decreases the amplitude of the prompt gravity perturbation at Kamioka (Fig. 1a) from -23 to $-5 \mathrm{~nm} / \mathrm{s}^{2}$ (Kimura 2018). Our selection criterion based on the model of Harms et al. (2015) eventually corresponded to the station selection with very low noise level and a hypocentral distance longer than $500 \mathrm{~km}$. 
For these 27 records, we applied a polarity reversal correction, i.e., the data was multiplied by -1 at stations where the predicted gravity change $\left(\delta g^{\mathrm{H}}\right)_{z}$ is positive, and vice versa, based on the model by Harms et al. (2015) (Fig. 1b). The predicted polarities at the 27 stations were the same; we just added them to obtain the stacked waveform.

For the stacking of Hi-net data, the trace selection criterion and a polarity reversal correction were based on Harms et al. (2015) as well. Because of the noisy data, traces were chosen based on the criterion of the $A_{S} / A_{\mathrm{N}}$ ratio being greater than unity. Note that the model of Harms et al. (2015) can provide a prompt gravity change for all three vector components.

\section{Appendix 4: Expression for theoretical gravito-gradiograms}

Here, we show the theoretical gravito-gradiogram, the waveform of a gravity gradient, in an explicit form. Our formula can be used to synthesize template waveforms for the detection of prompt gravity perturbations through the measurement of a gravity gradient or strain acceleration using state-of-the-art devices such as TOBA. Our expression is an extension of Harms et al. (2015) as it can deal with the general seismic source represented by a moment tensor.

We assume the same simplifications of Harms et al. (2015). The derivation starts from the equivalence between two potentials as follows (Harms et al. 2015):

$$
\delta \psi(\boldsymbol{x}, t)=-4 \pi G \rho_{0} \phi(\boldsymbol{x}, t),
$$

where $\boldsymbol{x}$ is the receiver position, $t$ the time, $G$ the gravitational constant, $\rho_{0}$ the density of the medium, $\delta \psi$ the gravity potential perturbation, and $\phi$ the compressional seismic potential. From Eq. 1, the gravity perturbation vector $\delta \boldsymbol{g}(\boldsymbol{x}, t)$ is represented as follows:

$$
\delta \boldsymbol{g}(\boldsymbol{x}, t)=-\nabla \delta \psi(\boldsymbol{x}, t)=4 \pi G \rho_{0} \nabla \phi(\boldsymbol{x}, t)=4 \pi G \rho_{0} \boldsymbol{u}^{\phi}(\boldsymbol{x}, t),
$$

where $\boldsymbol{u}^{\phi}$ is the scalar potential component of the seismic displacement $\boldsymbol{u}\left(\boldsymbol{u}^{\phi}=\nabla \phi\right)$. Employing the well-known solution of the seismic displacement from a general seismic source represented by a moment tensor (Aki and Richards 2002), we obtained the analytical expression for the components of the prompt gravity perturbations $\delta \boldsymbol{g}(\boldsymbol{x}, t)$ as follows:

$$
\begin{aligned}
\delta g_{n}=- & \left(15 \gamma_{n} \gamma_{p} \gamma_{q}-3 \gamma_{n} \delta_{p q}-3 \gamma_{p} \delta_{q n}-3 \gamma_{q} \delta_{n p}\right) \\
& \frac{G}{r^{4}} \int_{0}^{r / \alpha} \tau M_{p q}(t-\tau) \mathrm{d} \tau \\
+ & \left(6 \gamma_{n} \gamma_{p} \gamma_{q}-\gamma_{n} \delta_{p q}-\gamma_{p} \delta_{q n}-\gamma_{q} \delta_{n p}\right) \\
& \frac{G}{\alpha^{2} r^{2}} M_{p q}\left(t-\frac{r}{\alpha}\right) \\
+ & \gamma_{n} \gamma_{p} \gamma_{q} \frac{G}{\alpha^{3} r} \dot{M}_{p q}\left(t-\frac{r}{\alpha}\right),
\end{aligned}
$$

where $\gamma_{i}$ is the directional cosine, $\delta_{i j}$ the Kronecker delta, $r$ the distance between the source and receiver, $\alpha$ the P-wave velocity, and $M_{p q}(t)$ the moment function. This expression uses orthonormal bases and is familiar to seismologists. The first term on the right-hand side shows the prompt term. It coincides with that of Harms et al. (2015) for a shear dislocation (a double couple) source.

In contrast to the prompt gravity acceleration, the measurement of the corresponding prompt gravity gradient change (or strain acceleration) is not affected by the ground motion. It is expressed as the spatial derivative of Eq. 3 as follows:

$$
\begin{aligned}
\ddot{h}_{n m}:= & \frac{\partial \delta g_{n}}{\partial x_{m}}=4 \pi G \rho_{0} \frac{\partial u_{n}^{\phi}}{\partial x_{m}} \\
= & R_{5} \frac{G}{r^{5}} \int_{0}^{\frac{r}{\alpha}} \tau M_{p q}(t-\tau) d \tau+R_{3} \frac{G}{\alpha^{2} r^{3}} M_{p q}\left(t-\frac{r}{\alpha}\right) \\
& +R_{2} \frac{G}{\alpha^{3} r^{2}} \dot{M}_{p q}\left(t-\frac{r}{\alpha}\right)+R_{1} \frac{G}{\alpha^{4} r} \ddot{M}_{p q}\left(t-\frac{r}{\alpha}\right),
\end{aligned}
$$

where $\ddot{h}_{n m}$ denotes the $n m$ th component of the gravity gradient tensor and

$$
\begin{aligned}
R_{5}= & 105 \gamma_{n} \gamma_{p} \gamma_{q} \gamma_{m}-15\left(\delta_{m n} \gamma_{p} \gamma_{q}+\delta_{m p} \gamma_{q} \gamma_{n}\right. \\
& \left.+\delta_{m q} \gamma_{n} \gamma_{p}+\delta_{p q} \gamma_{n} \gamma_{m}+\delta_{q n} \gamma_{p} \gamma_{m}+\delta_{n p} \gamma_{q} \gamma_{m}\right) \\
& +3\left(\delta_{p q} \delta_{m n}+\delta_{q n} \delta_{p m}+\delta_{n p} \delta_{q m}\right), \\
R_{3}= & -45 \gamma_{n} \gamma_{p} \gamma_{q} \gamma_{m}+6\left(\delta_{m n} \gamma_{p} \gamma_{q}+\delta_{m p} \gamma_{q} \gamma_{n}\right. \\
& \left.+\delta_{m q} \gamma_{n} \gamma_{p}+\delta_{p q} \gamma_{n} \gamma_{m}+\delta_{q n} \gamma_{p} \gamma_{m}+\delta_{n p} \gamma_{q} \gamma_{m}\right) \\
& -\left(\delta_{p q} \delta_{m n}+\delta_{q n} \delta_{p m}+\delta_{n p} \delta_{q m}\right), \\
R_{2}= & -10 \gamma_{n} \gamma_{p} \gamma_{q} \gamma_{m}+\left(\delta_{m n} \gamma_{p} \gamma_{q}+\delta_{m p} \gamma_{q} \gamma_{n}\right. \\
& \left.+\delta_{m q} \gamma_{n} \gamma_{p}+\delta_{p q} \gamma_{n} \gamma_{m}+\delta_{q n} \gamma_{p} \gamma_{m}+\delta_{n p} \gamma_{q} \gamma_{m}\right), \\
R_{1}= & -\gamma_{n} \gamma_{p} \gamma_{q} \gamma_{m} .
\end{aligned}
$$

This tensor is symmetric and has six different components. Equation 4 has four terms on the right-hand side, and the first term is the prompt term. Each term consists of a (1) radiation pattern, (2) distance-dependent term, and (3) time-dependent term. Once we specify the moment tensor, theoretical waveforms for any receiver position can be efficiently calculated using the formula. The full expression of $\frac{\partial u_{n}}{\partial x_{m}}$ is presented in Kimura and Kame (2019). The expression of theoretical gravitogradiograms from a single-force source is presented in Kimura (2018). 
Received: 14 September 2018 Accepted: 19 February 2019

Published online: 11 March 2019

\section{Publisher's Note}

Springer Nature remains neutral with regard to jurisdictional claims in published maps and institutional affiliations.

\section{References}

Abbott BP et al (2016) Observation of gravitational waves from a binary black hole merger. Phys Rev Lett 116(6):61102. https://doi.org/10.1103/PhysR evLett.116.061102

Aki K, Richards PG (2002) Quantitative seismology, 2nd edn. University Science Books, Susalito

Ando M, Ishidoshiro K, Yamamoto K, Yagi K, Kokuyama W, Tsubono K, Takamori A (2010) Torsion-bar antenna for low-frequency gravitational-wave observations. Phys Rev Lett 105(16):161101. https://doi.org/10.1103/ PhysRevLett.105.161101

Goldstein P, Snoke A (2005) SAC availability for the IRIS community. IRIS DMC Newslett 7(1)

Harms J (2016) Transient gravity perturbations from a double-couple in a homogeneous half-space. Geophys J Int 205(2):1153-1164. https://doi. org/10.1093/gji/ggw076

Harms J, Ampuero JP, Barsuglia M, Chassande-Mottin E, Montagner J-P, Somala SN, Whiting BF (2015) Transient gravity perturbations induced by earthquake rupture. Geophys J Int 201(3):1416-1425. https://doi.org/10.1093/ gji/ggv090

Heaton TH (2017) Correspondence: response of a gravimeter to an instantaneous step in gravity. Nat Commun 8:66. https://doi.org/10.1038/s4146 7-017-01348-z

Imanishi Y (2001) Development of a high-rate and high-resolution data acquisition system based on a real-time operating system. J Geod Soc Jpn 47(1):52-57. https://doi.org/10.11366/sokuchi1954.47.52

Imanishi Y (2005) On the possible cause of long period instrumental noise (parasitic mode) of a superconducting gravimeter. J Geod 78:683-690. https://doi.org/10.1007/s00190-005-0434-5
Imanishi Y (2009) High-frequency parasitic modes of superconducting gravimeters. J Geod 83:455-467. https://doi.org/10.1007/s00190-008-0253-6

Juhel K, Ampuero JP, Barsuglia M, Bernard P, Chassande-Mottin E, Fiorucci D, Harms J, Montagner J-P, Vallée M, Whiting BF (2018) Earthquake early warning using future generation gravity strainmeters. J Geophys Res Solid Earth 123(12):10889-10902. https://doi.org/10.1029/2018JB016698 Juhel K, Montagner J-P, Vallée M, Ampuero JP, Barsuglia M, Bernard P, Clévédé E, Harms J, Whiting BF (2019) Normal mode simulation of prompt elastogravity signals induced by an earthquake rupture. Geophys J Int 216(2):935-947. https://doi.org/10.1093/gji/ggy436

Kimura M (2018) No identification of predicted earthquake-induced prompt gravity signals in data recorded by gravimeters, seismometers, and tiltmeters and its interpretation based on the principle of gravimetry. Master thesis, The University of Tokyo, Japan. https://repository.dl.itc.u-tokyo.ac jp/?action=pages_view_main\&active_action=repository_view_main_ item_detail\&item_id=51237\&item_no=1\&page_id=28\&block_id=31. Accessed 25 Feb 2019

Kimura M, Kame N (2019) Representation theorem and Green's function (3) - strain, stress, and density perturbation fields due to a point source using 2nd derivative of Green's function in an unbounded homogeneous isotropic elastic medium - Zisin 2(71):153-160. https://doi.org/10.4294/ zisin.2017-20 (in Japanese)

Montagner J-P, Juhel K, Barsuglia M, Ampuero JP, Chassande-Mottin E, Harms J, Whiting $B$, Bernard $P$, Clévédé $E$, Lognonné $P$ (2016) Prompt gravity signal induced by the 2011 Tohoku-Oki earthquake. Nat Commun 7:13349. https://doi.org/10.1038/ncomms13349

Shoda A, Ando M, Ishidoshiro K, Okada K, Kokuyama W, Aso Y, Tsubono K (2014) Search for a stochastic gravitational-wave background using a pair of torsion-bar antennas. Phys Rev D 89(2):27101. https://doi.org/10.1103/ PhysRevD.89.027101

Tonegawa T, Hirahara K, Shibutani T, Shiomi K (2006) Upper mantle imaging beneath the Japan Islands by Hi-net tiltmeter recordings. Earth Planets Space 58(8):1007-1012. https://doi.org/10.1186/BF03352605

Vallée M, Ampuero JP, Juhel K, Bernard P, Montagner J-P, Barsuglia M (2017) Observations and modeling of the elastogravity signals preceding direct seismic waves. Science 358:1164-1168. https://doi.org/10.1126/scien ce.aao0746

\section{Submit your manuscript to a SpringerOpen ${ }^{\odot}$ journal and benefit from:}

- Convenient online submission

- Rigorous peer review

- Open access: articles freely available online

- High visibility within the field

- Retaining the copyright to your article

Submit your next manuscript at $\boldsymbol{\nabla}$ springeropen.com 